\title{
Practical synthetic strategies towards lipophilic 6-iodotetrahydroquinolines and -dihydroquinolines
}

\author{
David R. Chisholm ${ }^{1}$, Garr-Layy Zhou ${ }^{1}$, Ehmke Pohl ${ }^{1,2}$, Roy Valentine ${ }^{3}$ \\ and Andrew Whiting ${ }^{* 1, \S}$
}

\author{
Full Research Paper \\ Address: \\ ${ }^{1}$ Centre for Sustainable Chemical Processes, Department of \\ Chemistry, Durham University, South Road, Durham, DH1 3LE, UK, \\ ${ }^{2}$ Biophysical Sciences Institute, Durham University, South Road, \\ Durham, DH1 3LE, UK and ${ }^{3}$ High Force Research Limited, Bowburn \\ North Industrial Estate, Bowburn, Durham, DH6 5PF, UK \\ Email: \\ Andrew Whiting* - andy.whiting@durham.ac.uk \\ * Corresponding author \\ § Fax: +44 (0)191384 4737; Tel: +44 (0)191334 2081 \\ Keywords: \\ cyclisation; dihydroquinoline; elimination; reduction; \\ tetrahydroquinoline \\ Beilstein J. Org. Chem. 2016, 12, 1851-1862. \\ doi:10.3762/bjoc. 12.174 \\ Received: 27 April 2016 \\ Accepted: 12 July 2016 \\ Published: 16 August 2016 \\ Associate Editor: J. P. Wolfe \\ (C) 2016 Chisholm et al.; licensee Beilstein-Institut. \\ License and terms: see end of document.
}

\begin{abstract}
The synthesis of novel tetrahydroquinolines (THQ) and dihydroquinolines (DHQ) are reported using three practical, scalable synthetic approaches to access highly lipophilic analogues bearing a 6-iodo substituent, each with a different means of cyclisation. A versatile and stable quinolin-2-one intermediate was identified, which could be reduced to the corresponding THQ with borane reagents, or to the DHQ with diisobutylaluminium hydride via a novel elimination that is more favourable at higher temperatures. Coupling these strongly electron-donating scaffolds to electron-accepting moieties caused the resulting structures to exhibit strong fluorescence.
\end{abstract}

\section{Introduction}

Tetrahydroquinolines (THQ) and dihydroquinolines (DHQ) are heterocyclic scaffolds that are ubiquitous in natural products, therapeutics, fluorophores and dyes [1]. Both are structures of great versatility, and their physical and chemical properties can be finely tuned using synthetic chemistry. Methods for their synthesis have been well studied, and range from the classic Skraup-Doebner-von Miller syntheses, to catalytic and asymmetric methods [2-4].
We required a straightforward synthesis of THQs and DHQs for the synthesis of a library of biocompatible fluorophores with the potential to be used in fluorescence microscopy applications. The desired intermediates were to be highly lipophilic, derivatisable and easy to synthesise on both small and multigram scales. A scaffold of general type 1 (Figure 1) was chosen as a synthetic target that could be $N$-alkylated with bulky alkyl groups for increased lipophilicity. An iodo substituent in the 


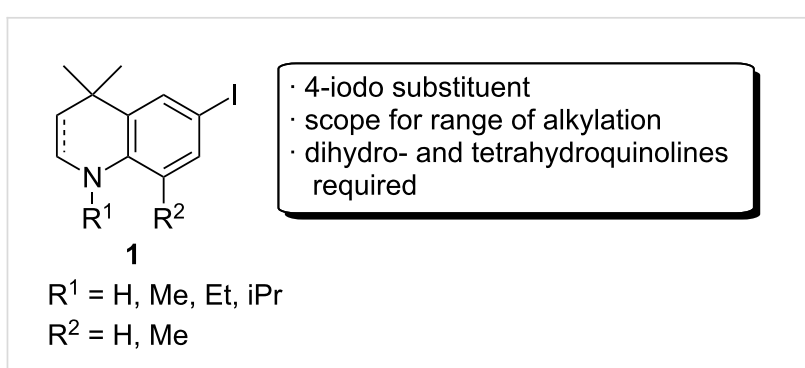

Figure 1: Tetrahydroquinoline (THQ) and dihydroquinoline (DHQ) scaffolds to be synthesised.

para-position relative to nitrogen (the 6-position) was required for further functionalisation by typical cross-coupling reactions.

THQ scaffolds similar to $\mathbf{1}$ are known in the literature, and have been typically prepared by two approaches. The first involves the alkylation of the starting aniline with the inexpensive 3,3dimethylallyl bromide/chloride, followed by cyclisation mediated by Lewis acids, or under acidic conditions [5,6]. A second, analogous approach involves an initial acylation with the commercially available 3,3-dimethylacryloyl chloride, followed by Friedel-Crafts cyclisations that also use Lewis acids [7]. The resultant quinolin-2-one is then reduced using strong hydride reducing agents such as $\mathrm{LiAlH}_{4}$ [8]. Similar THQs have also been prepared by a reductive Beckmann rearrangement of an oxime using diisobutylaluminium hydride (DIBAL) [9]. In contrast to this variety, the corresponding 1,4-DHQ of scaffold $\mathbf{1}$ has not been synthesised to our knowledge. Similar DHQs are typically prepared by methods such as ring-closing metathesis, or by controlled Birch-type reductions of the corresponding quinoline [10-12].

Very few instances of iodine-containing THQs or DHQs exist in the literature [13]. An initial investigation with the corresponding bromides indicated that these were unreactive towards many cross-coupling methodologies, presumably due to the highly electron-rich arene [14]. Reactions such as the Sonogashira coupling, for example, often required high catalyst loadings, phosphine additives and strong heating or microwave conditions in order to effect coupling with most acetylenes. Accordingly, it was anticipated that the aryl iodides would be more versatile due to their greater propensity to undergo oxidative additions.

Therefore, in this paper we report herein the development of facile, scalable and practical syntheses for 6-iodo-THQ and 6-iodo-DHQ scaffolds that can be employed in a variety of cross-coupling and derivatisation reactions. Three synthetic routes are described, each of which involves a different means of cyclisation to form the heterocyclic structure. We also describe a novel formation of the 1,4-DHQ of scaffold 1; a reaction that appears to be favoured both by the use of hydride reducing agents that produce stable tetrahedral intermediates and the high temperature collapse of these intermediates.

\section{Results and Discussion}

The aim of this investigation was to synthesise THQs and DHQs of the general scaffold 1 for the use in a variety of crosscoupling reactions, thus necessitating the presence of a halogen or pseudohalogen in the para-position relative to nitrogen. Most of the relevant literature focuses on the synthesis of the corresponding aryl bromides or aryl chlorides, but initial investigation with 6-bromo-THQs indicated that these were remarkably unreactive in cross-coupling reactions [14], and a reliable and scalable synthesis of the likely more reactive iodides was, therefore, sought.

We first considered the retrosynthetic analysis shown in Scheme 1, which requires cyclisation from the alkylated $N$-allylaniline 4 . The use of polyphosphoric acid (PPA) in cyclisation reactions is well known in the literature, and has been used to form a large variety of ring structures [15]. Iodination of 3 could then be realised by utilising the rich iodination literature available $[16,17]$.

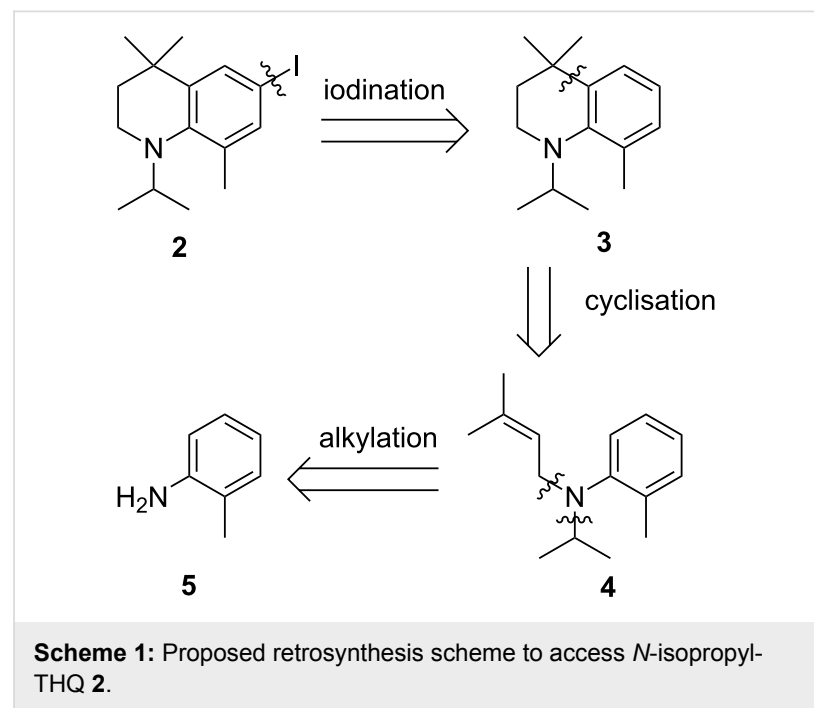

Isopropylation of the inexpensive $o$-toluidine (5) can be conducted by simply adding $\mathrm{NaOH}$ and 2-iodopropane to a refluxing solution of the aniline (Scheme 2). While this straightforward procedure was well suited to larger scale, some bisalkylation was difficult to avoid. However, the desired product 6 can be isolated using basic alumina chromatography. Reductive amination with acetone, mediated by $\mathrm{AcOH}$ and $\mathrm{NaOAc}$ was also pursued as an alternative strategy [18]. While being much easier to purify due to the elimination of the bis-alkyl- 

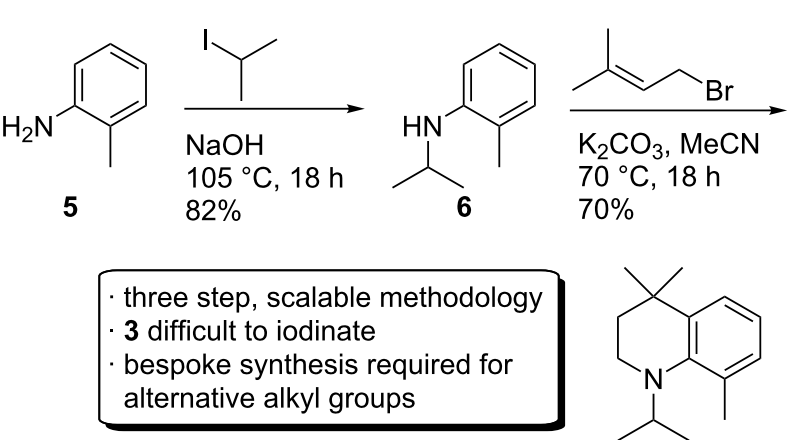<smiles>CC(C)=CCN(c1ccccc1C)C(C)C</smiles>

$$
\begin{aligned}
& 3 \text { difficult to iodinate } \\
& \text { bespoke synthesis required for } \\
& \text { alternative alkyl groups }
\end{aligned}
$$

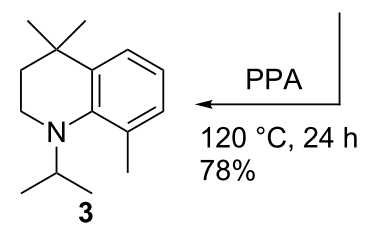

Scheme 2: Synthesis of THQ 3 by initial $N$-alkylations, followed by PPA-mediated cyclisation.

ation product, the yield of this approach was much more variable at larger scales.

Further $N$-alkylation with 3,3-dimethylallyl bromide was conducted using $\mathrm{K}_{2} \mathrm{CO}_{3}$. The resulting alkylated compound 4 was easily isolated by chromatography in good yield, however, the reaction was found to be difficult to force to completion, presumably due to steric hindrance. Cyclisation of 4 to the THQ 3 was realised by heating a PPA mixture of 4 to $120^{\circ} \mathrm{C}$.

Iodination of $\mathbf{3}$ using a variety of reagents including $\mathrm{I}_{2} / \mathrm{HgO}$ [19], ICl/AcOH, $N$-iodosuccinimide/TFA [20], $\mathrm{KI} / \mathrm{KIO}_{3} / \mathrm{AcOH}$ [21], and $N$-chlorosuccinimide/ $\mathrm{NaI} / \mathrm{AcOH}$ [22], all gave poor yields (0-30\%) according to GC-MS analysis, and the iodinated product 2 was difficult to isolate by chromatography. In light of these results, bromination was conducted with the aim of synthesising the corresponding aryl iodide $\mathbf{2}$ by halogen exchange (Scheme 3).<smiles>Cc1cccc2c1N(C(C)C)CCC2(C)C</smiles>

3

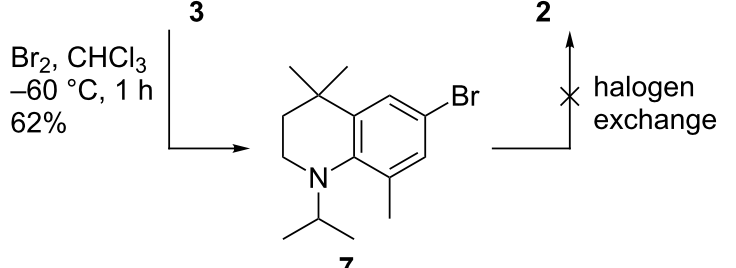

7

Scheme 3: Bromination of $\mathbf{3}$ and attempted halogen exchange of the intermediate 7 .

After careful optimisation of the reaction conditions, the bromide 7 was exclusively obtained by addition of 0.95 equiva- lents of bromine to a solution of $\mathbf{3}$ in chloroform maintained at $-60{ }^{\circ} \mathrm{C}$. However, halogen exchange under literature conditions was sluggish and even after 3 days only around $30 \%$ of the starting material had converted [23].

It was theorised that the low reactivity of $\mathbf{3}$ towards electrophilic aromatic iodination was caused by distortion of the THQ ring structure due to the need to minimise steric interactions between the $N$-iPr group and the neighbouring methyl group. This may result in poorer orbital overlap between the nitrogen lone pair and the aromatic $\pi$-system, thus reducing the $\mathrm{sp}^{2}$ character of the nitrogen, and therefore lowering the reactivity of the system towards electrophilic aromatic substitution. An alternative, analogous synthesis was accordingly devised, in which the unsubstituted THQ $\mathbf{1 0}$ was targeted, as outlined in Scheme 4 [24].

Reports of aza-Michael additions occurring in water indicated that the desired $\mathbf{8}$ could be synthesised under mild conditions, and we therefore decided to adapt these published conditions to larger scale work $[25,26]$. Initial attempts involving a 1:1 molar mixture of $o$-toluidine (5) and methyl vinyl ketone (MVK) indicated that only around $60 \%$ of the starting aniline had converted, particularly at larger scales $(>1 \mathrm{~g})$. Two equivalents of MVK were required to effect full conversion, however, under these conditions the bis-adduct was formed in around 6-10\% and this was difficult to remove by chromatography or distillation. Using one equivalent lowered the yield, but minimised bisadduct formation, which allowed facile purification by short path distillation on larger scales. Compound $\mathbf{8}$ was functionalised to the tertiary alcohol 9 by a Grignard reaction with $\mathrm{MeMgBr}$, which could be directly cyclised without purification to $\mathbf{1 0}$ by heating a DCM solution with a nominal amount of concentrated sulphuric acid. This procedure was much more straightforward when compared to the previous PPA reaction, and required a much less laborious work-up. 


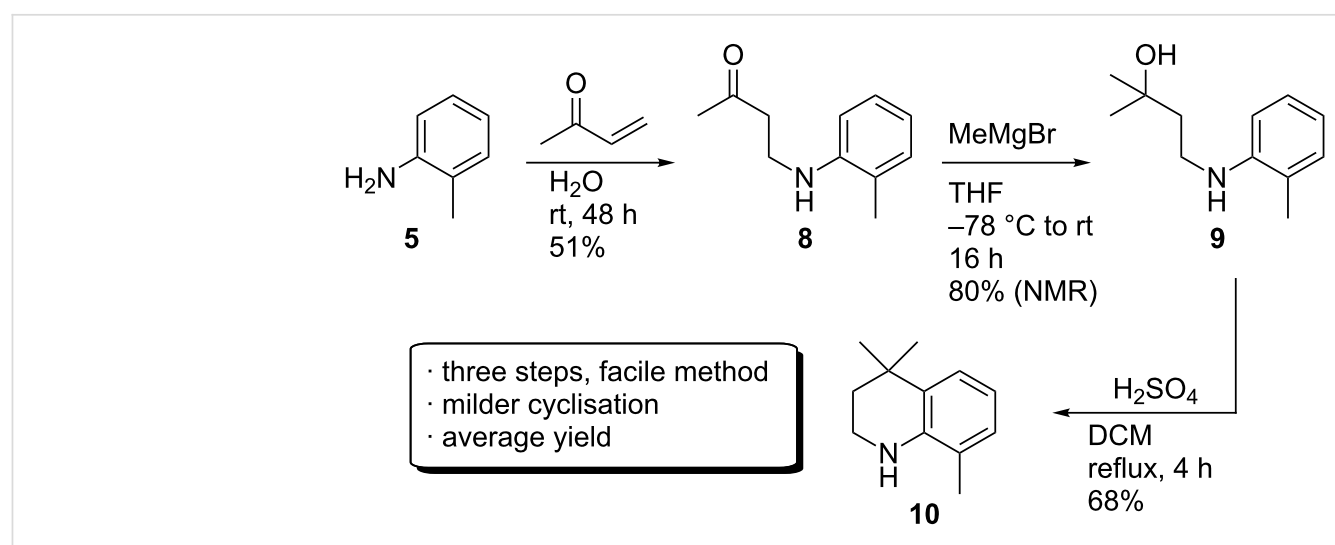

Scheme 4: Synthesis of THQ 10, by initial aza-Michael addition, followed by formation of the tertiary alcohol 9 , which was then cyclised with $\mathrm{H}_{2} \mathrm{SO}_{4}$.

The increased efficiency of this reaction can be attributed to improved orbital overlap. Protonation of the hydroxy group of 9 under the reaction conditions likely leads to the corresponding tertiary carbocation under equilibrium. The nitrogen lone pair can then assist the electrophilic cyclisation reaction, augmented by improved orbital overlap with the aromatic $\pi$-system, which in the case of 9 would be improved over $\mathbf{4}$ due to the lack of a second alkyl substituent and diminished steric repulsion that likely causes rotation of the nitrogen lone pair out of conjugation with the aromatic ring.

While the increased availability of the nitrogen lone pair appears to assist cyclisation in this system, iodination of $\mathbf{1 0}$ was still relatively low yielding, particularly with acidic iodination methods (presumably due to protonation and resultant deactivation of the nitrogen). Slightly higher yields were obtained with pyridine-iodine [16], however, the conversion was also low and isolation of the iodinated product was often difficult.

Despite the relative ease, the overall yield of this second route was considered too low to be a reliable source of THQs of the general scaffold 1. However, we were encouraged by the simplicity and ease of the $\mathrm{H}_{2} \mathrm{SO}_{4}$-mediated cyclisation reaction, and a third synthetic route was therefore devised that would employ this methodology.

A common approach in the literature towards similar THQs involves an initial acylation of the starting aniline using 3,3dimethylacryloyl chloride, followed by a high temperature cyclisation employing Lewis acids such as $\mathrm{AlCl}_{3}$ [7,27]. However, initial experiments indicated that under these cyclisation conditions, significant degradation and de-iodination occurred with iodinated intermediates. It was anticipated that the milder $\mathrm{H}_{2} \mathrm{SO}_{4}$ conditions could affect the cyclisation without these side reactions. To probe this, commercially available 4-iodo-2methylaniline (11) was employed as the starting material in order to circumvent the low-yielding iodination step. This starting material is also readily synthesised from $o$-toluidine (5) using a pyridine-iodine iodination [16].

Initial acylation of $\mathbf{1 1}$ with 3,3-dimethylacryloyl chloride and pyridine provided 12 in good yield (Scheme 5). The acid-catalysed reaction with 12 was predicted to proceed via initial formation of the corresponding tertiary alcohol involving a Markovnikov addition, before cyclisation as with 10. Indeed, cyclisation product 13 (see Supporting Information File 1 for crystal structure) was produced cleanly in a $67 \%$ yield, which was easily separated chromatographically from the remaining starting material. However, the reaction occurred at a significantly slower rate than with $\mathbf{9}$; particularly on larger reaction scales where complete conversion of the starting material required long reaction times ( $>48 \mathrm{~h}$ ). The significantly slower rate is likely due to the reduced availability of the nitrogen lone pair in 12. Favourably, however, no signs of de-iodination or degradation were observed under these cyclisation conditions. Cyclisation product 13 was readily reduced to the iodinated THQ 14 (see Supporting Information File 1 for crystal structure) using borane-dimethyl sulphide complex. A slight molar excess of the reducing agent causes de-iodination to give $\mathbf{1 0}$ in small quantities.

$N$-Alkylation with highly lipophilic alkyl groups such as an isopropyl was next considered (Scheme 6). The quinolin-2-one 13 was predicted to be a more amenable alkylation partner than the THQ 14 due to the likely lower $\mathrm{p} K_{\mathrm{a}}$ of the amide proton. To assess this, 13 was reacted with $\mathrm{NaH}$ and 2-iodopropane in DMF at $80{ }^{\circ} \mathrm{C}$ overnight. However, 13 displayed a marked lack of reactivity towards isopropylation, and only around $40 \%$ of the starting material 13 was converted. Interestingly, the major product from the reaction was the $O$-iPr imine $\mathbf{1 5 b}$ as indicated by a low field ${ }^{1} \mathrm{H}$ chemical shift of the isopropyl proton (5.39 ppm), and later confirmed by X-ray crystallography (the 


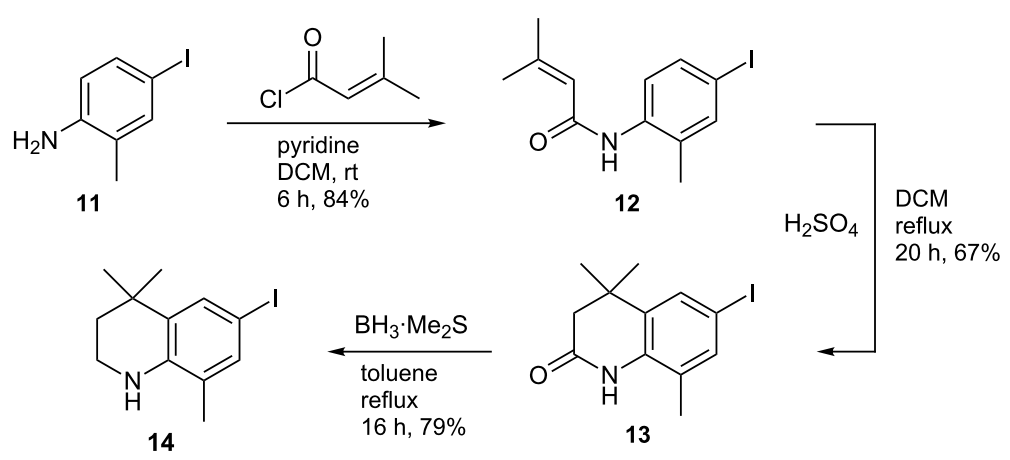

14

$$
\begin{aligned}
& \text { straighforward method and purification } \\
& \text { cyclisation much slower at larger scales } \\
& \text { - average to good yields } \\
& \text { facile reduction to THQ } 14
\end{aligned}
$$<smiles>Cc1cc(I)cc2c1NC(=O)CC2(C)C</smiles>

13<smiles>Cc1cc(I)cc2c1NCCC2(C)C</smiles>

14

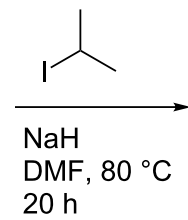

DMF

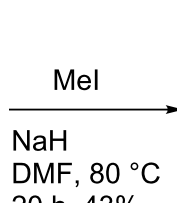

20 h, $43 \%$<smiles>Cc1cc(I)cc2c1N(C(C)C)C(=O)CC2(C)C</smiles>

$15 a, 3 \%$<smiles>Cc1cc(I)cc2c1N(C)CCC2(C)C</smiles>

16

Scheme 6: N-Alkylation of 13 and 14.

crystal structure is shown in Supporting Information File 1). The $N$-iPr product 15a was isolated in only $3 \%$ yield. This, therefore, indicates that the electrophile reacts faster with the oxide anion of 13. Repeating the reaction with 15-crown-5 to limit the effect of the sodium cation did not appreciably effect the product distribution. It would, therefore, appear that steric hindrance from the neighbouring methyl group is in fact the main determinant of the product distribution.

Alkylation of $\mathbf{1 4}$ was also marked by a general lack of reactivity; the methylated 16 was isolated in only a $43 \%$ yield. Ethylation was possible, albeit in much lower yield and only negligible amounts $(1-2 \%)$ of the iPr adduct were isolated. Alkylation using silver(I) oxide was also possible, but with similarly low yields.

The ortho-methyl group had originally been incorporated as protection to block the ortho-amino centre against oxidation
[14], and to increase lipophilicity. However, because the methyl group appeared likely to be the cause for the lack of reactivity of 13 and 14 towards alkylation, a synthesis of the corresponding quinolin-2-one derived from 4-iodoaniline (17) was pursued in order to increase the ease of alkylation.

The initial acylation of $\mathbf{1 7}$ (commercially available, or easily synthesised using literature methods [16]) proceeded well to give 18, and this could easily be applied to larger scale ( $>25 \mathrm{~g})$ synthesis. The acid-catalysed cyclisation again was much slower than for the synthesis of $\mathbf{1 0}$, and at large scale in particular; the reaction could take at least 48 hours for completion. An alternative large-scale protocol was, therefore, investigated. In contrast to the high temperature Lewis acid-mediated cyclisations reported in the literature [7], it was found that simply stirring a DCM solution of $\mathbf{1 8}$ with 1.5 equivalents of $\mathrm{AlCl}_{3}$ at room temperature for 2.5 hours gave the quinolin-2-one 19 in excellent yield after recrystallisation from EtOH (see Support- 
ing Information File 1 for the crystal structure). While facile, the reaction was highly dependent both on the number of equivalents of $\mathrm{AlCl}_{3}$ used and the reaction time. Leaving the reaction mixture to stir with 1.5 equivalents of $\mathrm{AlCl}_{3}$ for longer periods $(>3 \mathrm{~h})$ resulted in minor de-iodination, whereas less than 2 hours gave incomplete cyclisation. Using 2 equivalents of $\mathrm{AlCl}_{3}$ causes rapid cyclisation after 1 hour, but the de-iodinated form was also present. Using 1.25 equivalents appeared to inhibit de-iodination completely, but the cyclisation reaction tended to plateau before full completion.

Without the ortho-methyl, preference for isopropylation switched to $N$-alkylation, providing $50 \%$ of $20 \mathrm{a}$ and $26 \%$ of the $O$-isopropylimine 20b; a mixture that could be separated by chromatography. Full conversion of the starting material was difficult to achieve and appeared to plateau at around $75 \%$. However, the reaction was easily conducted and both products isolated on larger scale (30 g). Encouragingly, the iodine substituent was stable throughout the optimised synthesis of the quinolin-2-one intermediate (Scheme 7).
Reduction to the THQ 21 was easily conducted with borane-dimethyl sulphide complex as described previously, in excellent yield (Scheme 8). In addition, upon trialling different conditions for the reduction, $\mathrm{LiAlH}_{4}$ was found to afford a small amount (ca. 5\%) of 1,4-DHQ 22 in addition to 21 . Ab initio simulation of $\mathbf{2 2}$ (Figure 2) indicated that the enamine functionality causes a flattening of the dihydroquinoline ring to give a more planar, quinoline-like structure. We were intrigued by the result of these stereoelectronic and conformational

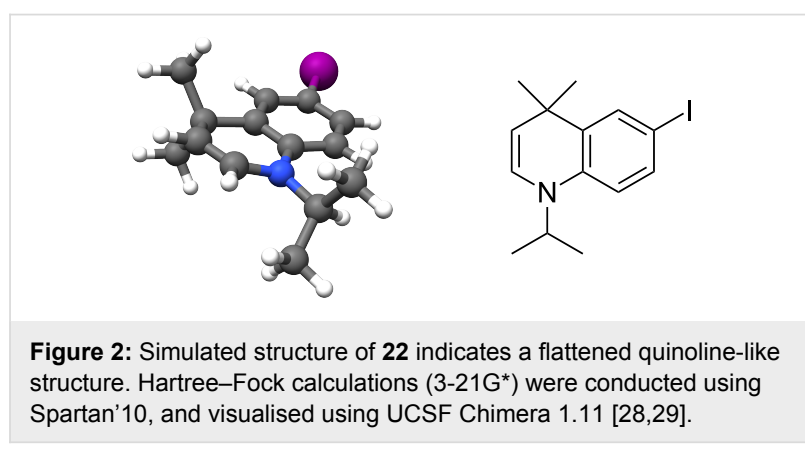

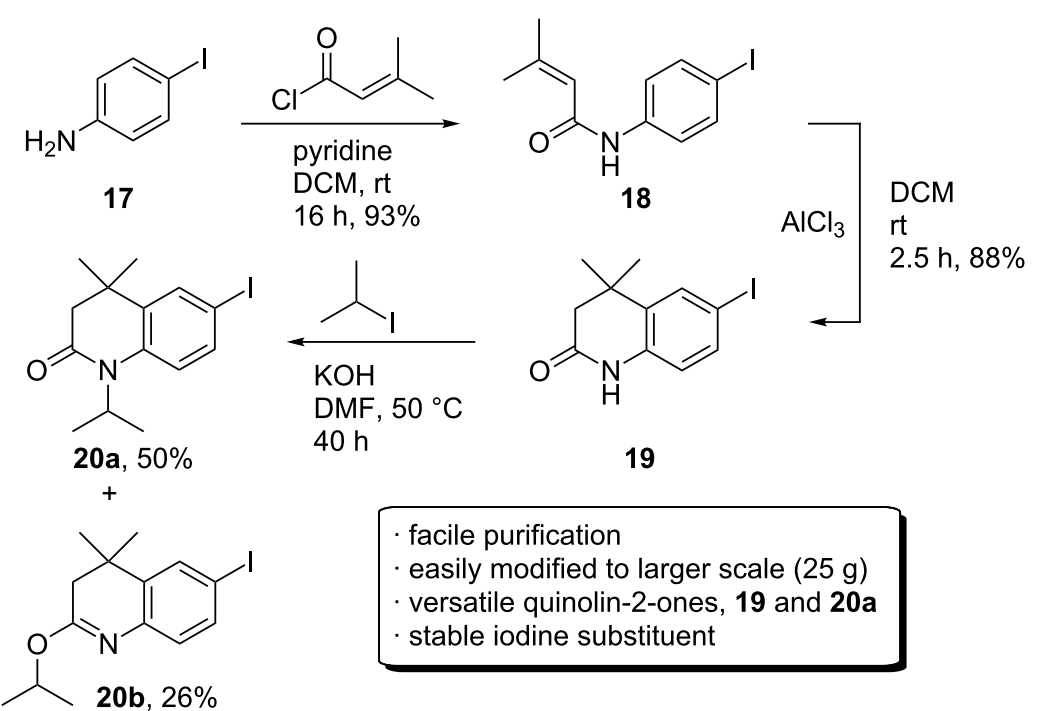

Scheme 7: Facile route for the synthesis of $20 a$.

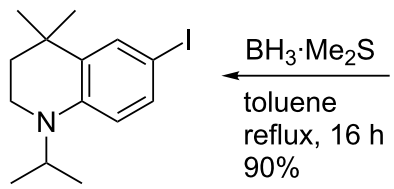

21<smiles>CC(C)N1C(=O)CC(C)(C)c2ccc(I)cc21</smiles>

$20 \mathrm{a}$

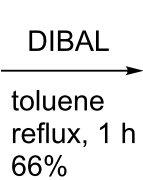

$66 \%$<smiles>CC(C)N1C=CC(C)(C)c2cc(I)ccc21</smiles>

22 
effects in terms of the chromophoric properties of the resulting systems and an improved synthesis of $\mathbf{2 2}$ was therefore pursued.

In light of its formation by $\mathrm{LiAlH}_{4}$, we anticipated that production of $\mathbf{2 2}$ would be favoured if the intermediate tetrahedral complex in the reduction reaction was more stable. An elimination reaction could then operate to give $\mathbf{2 2}$, either during aqueous work-up, or in situ collapse of the intermediate complex and subsequent reduction of the resulting iminium ion via elimination. As a Lewis acidic reducing agent, it was anticipated that DIBAL could be better suited to the reduction of the electron-rich amide of 20a, and DIBAL is also known to produce relatively stable tetrahedral intermediates [30]. Indeed, initial experiments using 1 equivalent of DIBAL in THF at reflux, with an acidic work-up, returned a $35 \%$ yield of $\mathbf{2 2}$ by ${ }^{1} \mathrm{H}$ NMR, along with $15 \%$ of $\mathbf{2 1}$, and unreacted starting material.

This result highlighted an apparent lack of reactivity in THF and the reaction was, therefore, monitored in THF, DCM and toluene using in situ FTIR spectroscopy (ReactIR). The amide carbonyl stretch of 20a was followed by IR during the addition of one equivalent of DIBAL at rt (see Supporting Information File 1 for details). The reactions in DCM and toluene indicated a rapid reduction of the amide carbonyl stretch. In contrast, the reaction in THF displayed a lower initial addition rate, presumably due to competitive coordination of DIBAL by the solvent, and appeared to plateau at a low conversion. A further addition of 1 equivalent of DIBAL did further consume the amide (shown by a further loss of the $\mathrm{C}=\mathrm{O}$ stretch by IR), however, again, progress appeared to plateau. This indicated that the reaction with the amide group was markedly faster in a non-polar solvent, and further work suggested that the yield of $\mathbf{2 2}$ was also increased under such conditions. Addition of DIBAL at lower temperatures $\left(0,-40\right.$, and $\left.-78{ }^{\circ} \mathrm{C}\right)$ was found to significantly slow the reaction in all solvents.

Further investigation also highlighted the interesting role of the temperature of the reaction mixture prior to the addition of DIBAL on the product distribution. Increased NMR yields of DHQ 22 relative to THQ 21 were recorded (as shown in Table 1), as the temperature of the 20a solution was increased prior to DIBAL addition. This trend indicates that $\mathbf{2 2}$ may be formed from in situ fragmentation of the intermediate DIBAL complex followed by an elimination process that is favoured at higher temperature, as postulated in Scheme 9.

Chromatographic separation of compounds $\mathbf{2 1}$ and $\mathbf{2 2}$ on silica resulted in co-elution; however, the two compounds could be separated on neutral alumina using a non-polar eluent. Selectivity for $\mathbf{2 2}$ on larger scales was slightly lower, though the
Table 1: Temperature and solvent effects from the addition of DIBAL to $20 a .^{a}$

\begin{tabular}{lllll} 
Solvent & Temp. $\left({ }^{\circ} \mathrm{C}\right)$ & $\mathbf{2 0 a}(\%)$ & $\mathbf{2 1}(\%)$ & $\mathbf{2 2}(\%)$ \\
\hline pentane & $\mathrm{rt}$ & 26 & 19 & 55 \\
cyclohexane & 60 & 2 & 14 & 79 \\
cyclohexane & 81 & 0 & 15 & 85 \\
toluene & 111 & 10 & 7 & 83 \\
xylenes & 140 & 13 & 4 & 83 \\
\hline
\end{tabular}

aOne equivalent of DIBAL (1.0 $\mathrm{M}$ in cyclohexane) added to preheated solution of 20a (ca. $0.25 \mathrm{mmol}$, in $4 \mathrm{~mL}$ ), stirred rapidly for $1 \mathrm{~h}$, quenched with $20 \%$ aq w/v NaOH, extracted in EtOAc, washed with $\mathrm{H}_{2} \mathrm{O}$ and brine, dried $\left(\mathrm{MgSO}_{4}\right)$ and evaporated. Yields of $\mathbf{2 1}$ and $\mathbf{2 2}$ estimated from ${ }^{1} \mathrm{H}$ NMR analysis of the crude mixture.

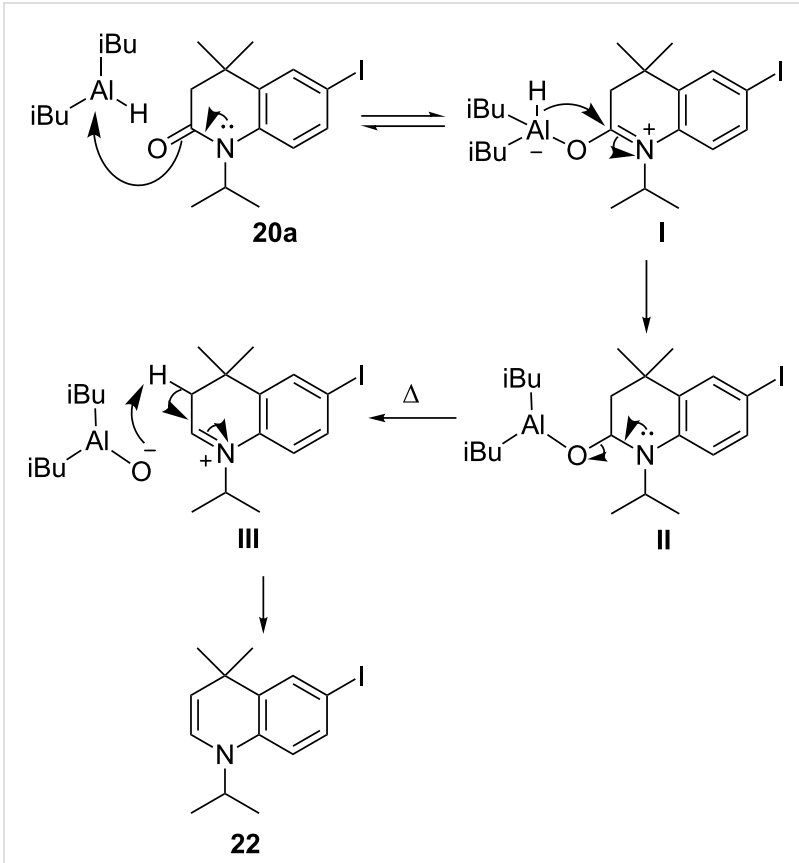

Scheme 9: Postulated mechanism for the formation of 22 using DIBAL.

DHQ was still isolated in a satisfactory $66 \%$ yield on one gram scale. Unlike borane, $\mathrm{LiAlH}_{4}$ and similar reagents, DIBAL did not cause de-iodination, even with larger excesses (up to 2 equivalents).

There are few references to this type of reaction in the literature, however, to our knowledge this is the only such reaction reported with an aromatic amide [31]. Organolithium reagents have been used to synthesise the analogous alkylated DHQ compounds [27].

Compounds $\mathbf{2 1}$ and $\mathbf{2 2}$ were found to undergo slow degradation (over $2-4$ weeks), as indicated by the observation that solutions 
in $\mathrm{CDCl}_{3}$ slowly turned pink (22 in particular). However, these compounds did not require special handling or precautions, and could be further derivatised without issue. In contrast, 20a was stable indefinitely when stored neat, or in solution at room temperature.

This interesting reaction was conducted with a variety of quinolin-2-ones bearing differing alkyl substituents in order to ascertain whether steric effects promote collapse of the intermediate aluminium complex via fragmentation/elimination, and to assess the generality of this approach. Table 2 highlights the effect of the presence of $N$-alkyl groups, and an aryl group in the benzylic position of the quinolin-2-one on the synthesis of the corresponding DHQs using the DIBAL reaction developed for the synthesis of $\mathbf{2 2}$.

Table 2: DIBAL reductions of quinolin-2-ones 23a-e using the optimised method to synthesise 22 . $^{a}$

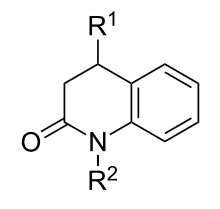

$23 a-e$

\section{DIBAL} toluene reflux<smiles>[R]C1CCN([R2])c2ccccc21</smiles>

$24 a-e$

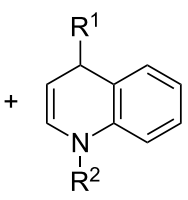

$25 a-e$

\begin{tabular}{llllll}
\hline Quinolin-2-one & $\mathrm{R}^{1}$ & $\mathrm{R}^{2}$ & $\mathbf{2 3}(\%)$ & $\mathbf{2 4}(\%)$ & $\mathbf{2 5}(\%)$ \\
\hline 23a & $\mathrm{Ph}$ & $\mathrm{H}$ & 0 & 100 & 0 \\
23b & $\mathrm{Ph}$ & $\mathrm{Me}$ & 0 & 38 & 62 \\
23c & $\mathrm{H}$ & $\mathrm{H}$ & 12 & 88 & 0 \\
23d & $\mathrm{H}$ & $\mathrm{Et}$ & $0^{\mathrm{b}}$ & $63^{\mathrm{b}}$ & $21^{\mathrm{b}}$ \\
23e & $\mathrm{H}$ & $\mathrm{Bn}$ & $0^{\mathrm{c}}$ & $35^{\mathrm{c}}$ & Trace $^{\mathrm{c}}$ \\
\hline
\end{tabular}

aDIBAL (1.0 M in toluene, 1.2 equivalents or 2.0 equivalents for $23 a$ and $\mathbf{2 3 c}$ ) added to a refluxing solution of quinolin-2-one $\mathbf{2 3 a - e}$ $(0.5-3.0 \mathrm{mmol})$ in toluene stirred rapidly at reflux until TLC analysis indicated completion. Then quenched with $20 \%$ aq w/v $\mathrm{NaOH}$, extracted with EtOAc, washed with $\mathrm{H}_{2} \mathrm{O}$ and brine, dried $\left(\mathrm{MgSO}_{4}\right)$ and evaporated. Yields of 23a-e, 24a-e and $\mathbf{2 5 a - e}$ were estimated from ${ }^{1} \mathrm{H}$ NMR analysis of the crude mixture (see Supporting Information File 1). ${ }^{b}$ A ring-opened ethylaniline ( $9 \%$ ) and another byproduct were also present $(7 \%)$. ${ }^{\mathrm{C}} \mathrm{A}$ ring-opened benzylaniline was also present $(61 \%)$.

The absence of an $\mathrm{N}$-alkyl group (23a and 23c) was found to completely disfavour the formation of the corresponding DHQ. Conducting these reactions with 1.2 equivalents of DIBAL resulted in low conversion (30-40\%) to the corresponding THQs (24a and 24c), presumably due to competitive deprotonation of the amide proton by DIBAL. When repeated with 2.0 equivalents, $\mathbf{2 4 a}$ and $\mathbf{2 4 c}$ were cleanly synthesised in $100 \%$ and $88 \%$ yields according to ${ }^{1} \mathrm{H}$ NMR analysis of the crude mixtures.

Addition of an $N$-alkyl group to supplement the steric effect of the benzylic phenyl $(N-\mathrm{Me}, \mathbf{2 3} \mathbf{b})$ resulted in the reaction favouring the production of the DHQ $\mathbf{2 5} \mathbf{b}$ (62\%) over the THQ 24b (38\%). Retaining the $N$-alkyl group whilst removing the steric effect of this benzylic phenyl group (23d) now switched the preference of the reaction towards the THQ 24d (63\%) compared to DHQ 25d (21\%). In contrast to the clean conversions of the other quinolin-2-ones, crude ${ }^{1} \mathrm{H}$ NMR analysis of the DIBAL reduction of $\mathbf{2 3 d}$ indicated a variety of side products, chief among which was the corresponding ring opened $N$-ethylaniline (9\%). Indeed, when moving to the $N$-benzylquinolin-2one $23 \mathbf{e}$, the major product was that of the ring opened $N$-benzylaniline (61\%) and was favoured over the THQ 24e (3\%) and only trace indications of DHQ 25e were apparent.

While these results indicated that this reaction was not completely general, comparison of the NMR yields began to provide further mechanistic insight. Clearly, Table 2 shows that an $N$-alkyl substituent is required in order to encourage formation of the DHQ. The presence of larger $N$-substituents (23d,e) did little to improve the yield of the corresponding DHQ over $\mathbf{2 3 b}$, thus indicating that the presence of an alkyl group simply retards reduction of the imine to the THQ, such that the competing elimination process can operate.

A clear increase in the yield of the DHQ is also observed upon addition of a steric influence in the benzylic position of the quinolin-2-one (20a/23b). Given the high temperature, it may be that 1,3-diaxial steric interactions between the benzylic substituent(s) and the intermediary iminium ion are such that a conformation needs to be adopted that both allows and indeed, favours the proposed elimination pathway, with the expelled DIBAL-derived aluminate likely functioning as the base.

With optimised syntheses of $\mathbf{2 1}$ and $\mathbf{2 2}$ in hand, the reactivity of their iodo substituents towards cross-coupling reactions was compared to that of the corresponding aryl bromides. Table 3 shows an example where 21 and 6-bromo-THQ 26 (synthesised using the optimised conditions for 21) were reacted under typical Suzuki conditions with boronic ester 27. Analysis of the crude ${ }^{1} \mathrm{H}$ NMR spectra for the reactions indicated full conversion of the 6-iodo-THQ 21, but moderate conversion (56\%) in the case of the 6-bromo-THQ 26. This highlights the greater propensity for $\mathbf{2 1}$ to undergo oxidative addition, due to the more reactive iodo-substituent. Further analysis indicated that biaryl 28 strongly absorbs light at $360 \mathrm{~nm}$, and when excited at this wavelength exhibited a red-shifted, intense, emission peaking at $450 \mathrm{~nm}$ (Figure 3), thus highlighting the chromophoric effect of attaching a conjugated electron acceptor to the strongly electron donating THQ moiety.

To enable a wider range of cross-coupling reactions and to compare their 3-dimensional structures, the corresponding 


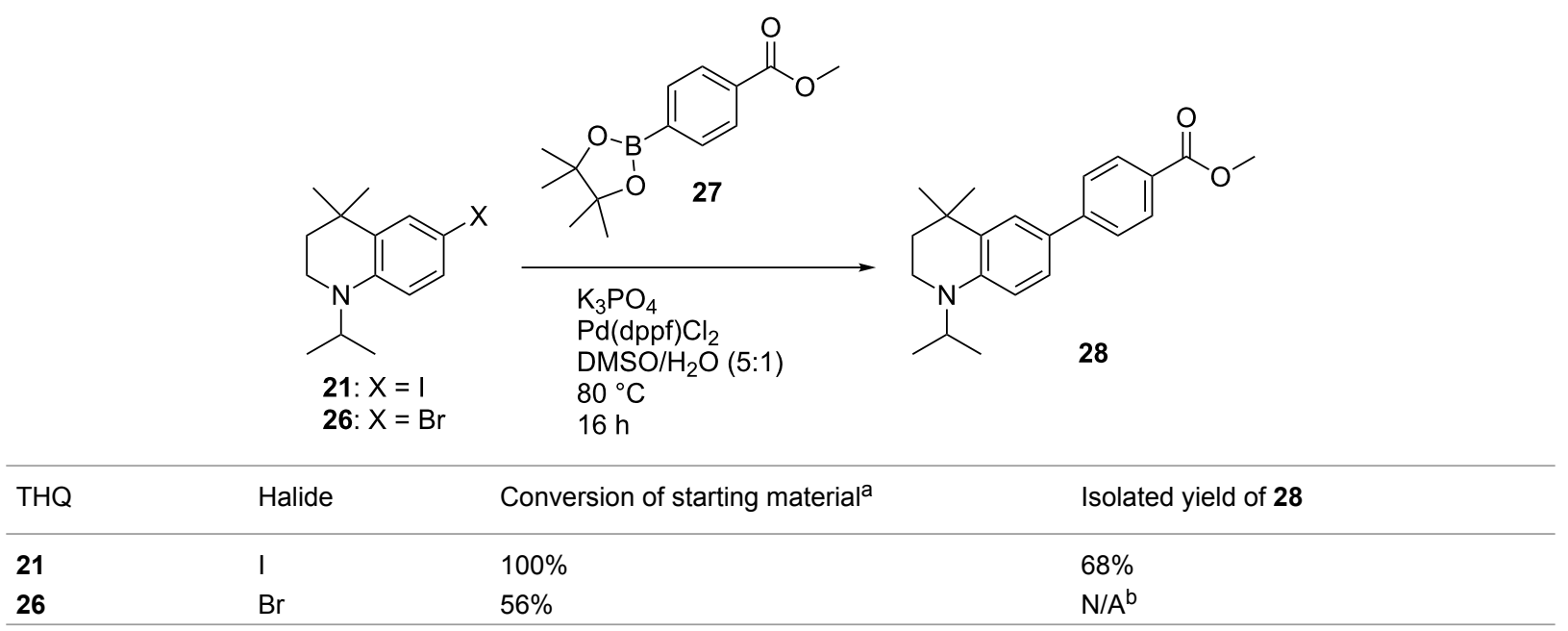

aConversion of the starting THQs $\mathbf{2 1 / 2 6}$ to the coupling product 28 was estimated by comparing the integrals of the $\mathrm{CH}_{2}$ adjacent to the nitrogen (the 2-position) in the crude ${ }^{1} \mathrm{H}$ NMR spectrum. ${ }^{b}$ Not isolated.

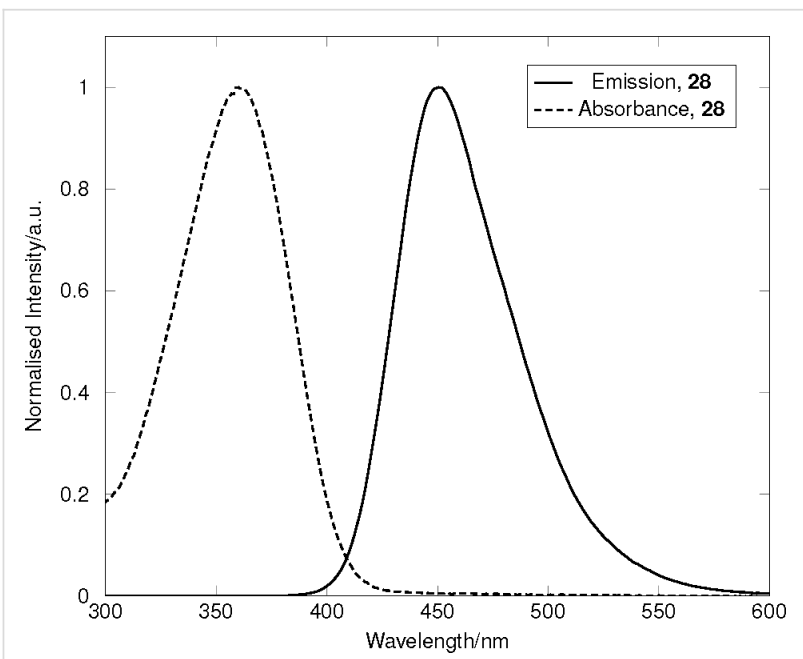

Figure 3: Combined, normalised absorption and emission spectra of 28 in chloroform. Absorption spectrum was recorded at $10 \mu \mathrm{M}$. Emission spectrum was recorded at $100 \mathrm{nM}$, with excitation at $360 \mathrm{~nm}$.

boronic esters 29 and $\mathbf{3 0}$ were prepared from $\mathbf{2 1}$ and $\mathbf{2 2}$ using typical Miyaura borylation conditions (Scheme 10) [32]. Both were highly crystalline compounds, and comparison of the two crystal structures (Figure 4) highlighted the more planar structure of $\mathbf{3 0}$ as a result of the enamine function, as predicted by ab initio methods in Figure 2. Furthermore, TLC samples of $\mathbf{3 0}$ were found to be fluorescent under a UV lamp, and an absorbance/emission analysis was accordingly conducted in diethyl ether and chloroform.

Figure 5 shows the combined, normalised absorbance and emission profiles in diethyl ether at $10 \mu \mathrm{M}$ and $100 \mathrm{nM}$, respective-

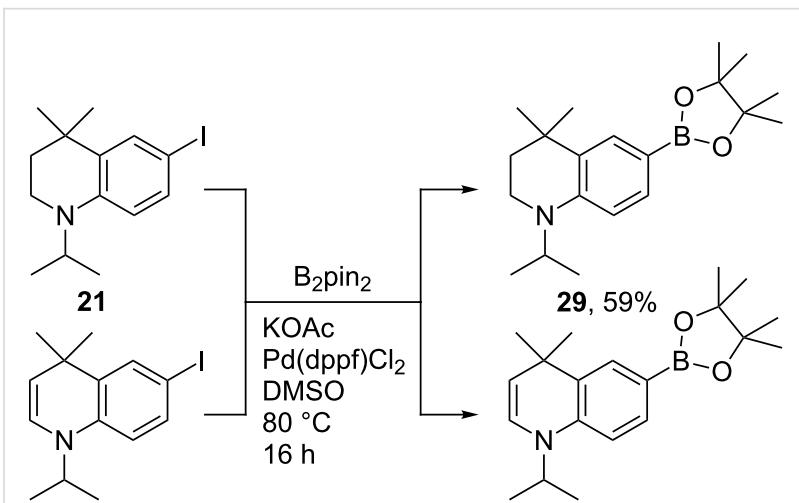

22

$30,53 \%$

Scheme 10: Miyaura borylation of 21 and 22 to give crystalline boronic esters 29 and 30 .

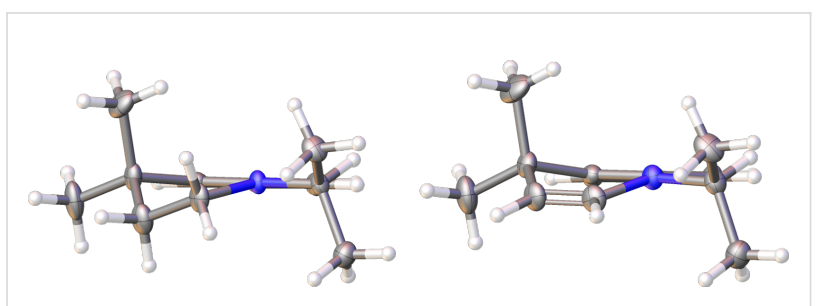

Figure 4: Comparison of the crystal structures of 29 (left) and $\mathbf{3 0}$ (right) as viewed along the plane of the aromatic ring, with the pinacolate ester groups removed for clarity. DHQ $\mathbf{3 0}$ exhibits a more planar structure. Compounds 29 and $\mathbf{3 0}$ were visualised using Olex2 [33].

ly. In diethyl ether, $\mathbf{3 0}$ exhibited a major absorbance at $318 \mathrm{~nm}$, and when excited at this wavelength, exhibited an emission that peaks at $370 \mathrm{~nm}$. A similar maximal absorbance was observed 
in chloroform $(323 \mathrm{~nm})$, and excitation at this wavelength produced an emission that was significantly lower in intensity, and mildly red shifted (to $376 \mathrm{~nm}$ ). This behaviour indicates that 1,4-DHQ structures of this type may function as more effective donor chromophores in dyes and fluorophores than the corresponding THQs due to improved orbital overlap between the nitrogen lone pair and the aromatic $\pi$-system by virtue of the more planar ring structure.

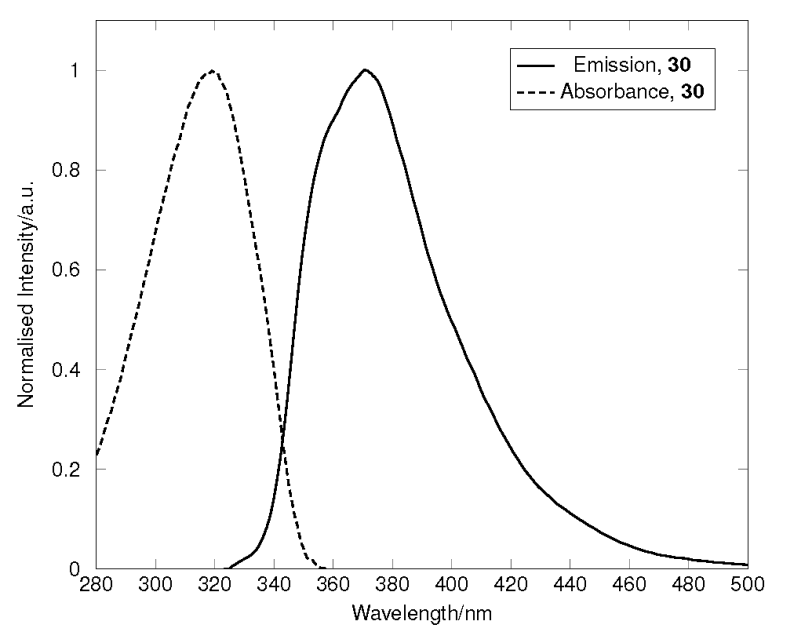

Figure 5: Combined, normalised absorption and emission spectra of 30 in diethyl ether. Absorption spectrum was recorded at $10 \mu \mathrm{M}$. Emission spectrum was recorded at $100 \mathrm{nM}$, with excitation at $318 \mathrm{~nm}$.

\section{Conclusion}

In conclusion, we have explored a wide range of methods for the synthesis of highly lipophilic tetrahydroquinolines and dihydroquinolines. Their iodo-substituents are reactive substrates for direct cross coupling using the plethora of cross-coupling methodologies in the literature. Iodoquinolin-2-ones 13, 19 and 20a, are stable intermediates, and we have developed straightforward, practical and scalable procedures towards their synthesis. We have also demonstrated the novel synthesis of dihydroquinoline 22, by what appears to be a collapse of the intermediate DIBAL complex that is favoured at higher temperatures. Further mechanistic insight into this interesting reaction was garnered by varying the structure of the quinolin-2-one starting material, and indicated that increased steric bulk in the benzylic position and an $\mathrm{N}$-alkyl substituent improves selectivity for the DHQ over the corresponding THQ. Comparison of the reactivity of 6-iodo-THQ 21 and 6-bromo-THQ 26 in a typical Suzuki coupling reaction showed significantly improved conversion in the case of the iodide. The resulting biaryl $\mathbf{2 8}$ was found to be highly fluorescent, highlighting the suitability of general structure $\mathbf{1}$ as an electron donor for the design of charge transfer fluorophores. Finally, crystallographic analysis of the boronic esters 29 and $\mathbf{3 0}$ highlighted a subtle flattening of the DHQ structure when compared to the saturated THQ; a structural characteristic that was found to cause fluorescence in 30, indicating that the DHQ may be a more effective electron donor than the THQ. We are currently utilising the optimised syntheses of these interesting compounds in a range of novel fluorophores, and their applications will be communicated in due course.

\section{Experimental Synthetic chemistry}

Reagents were purchased from Sigma-Aldrich, Acros Organics, Alfa-Aesar and Fluorochem and used without further purification unless otherwise stated. 4-Iodo-2-methylaniline and 4-iodoaniline were either purchased from Fluorochem or prepared according to a literature method [16]. Solvents were used as supplied, and dried before use if required with appropriate drying agents or using an Innovative Technologies Inc. Solvent Purification System. Thin-layer chromatography (TLC) was conducted using Merck Millipore silica gel 60G F254 25 glassplates and/or TLC-PET foils of aluminium oxide with fluorescent indicator $254 \mathrm{~nm}(40 \times 80 \mathrm{~mm})$ with visualisation by UV lamp or appropriate staining agents. Flash column chromatography was performed using $\mathrm{SiO}_{2}$ from Sigma-Aldrich (230-400 mesh, 40-63 $\mu \mathrm{m}, 60 \AA$ ), or activated neutral aluminium oxide (Alumina) from Sigma-Aldrich, and monitored using TLC. NMR spectra were recorded in $\mathrm{CDCl}_{3}$ using Varian VNMRS-700, Varian VNMRS-600, Bruker Avance-400 or Varian Mercury-400 spectrometers operating at ambient probe temperature. NMR peaks are reported as singlet (s), doublet (d), triplet (t), quartet (q), broad (br), septet (sept), combinations thereof, or as a multiplet (m). ESMS was performed by the Durham University departmental service using a TQD (Waters Ltd, UK) mass spectrometer with an Acquity UPLC (Waters Ltd, UK), and accurate mass measurements were obtained using a QtoF Premier mass spectrometer with an Acquity UPLC (Waters Ltd, UK). IR spectra were recorded using a Perkin Elmer FTIR spectrometer. Melting points were obtained using a Gallenkamp melting point apparatus and are uncorrected. Elemental analysis was conducted by the Durham University departmental service using an Exeter Analytical CE-440 analyser. UV-vis spectroscopy was conducted using a CARY100 UV-visible spectrophotometer using the Cary WinUV Scan software 3.00(182). Fluorescence emission spectroscopy was conducted using a Perkin Elmer LS 55 fluorescence spectrometer. All in situ FTIR spectroscopy experiments (ReactIR, Mettler Toledo) were carried out using a ReactIR 15 with MCT detector. Apodization $=$ Happ General. Probe: Prob A DiComp (Diamond) connected via KAgX $9.5 \mathrm{~mm} \times 2 \mathrm{~m}$ Fiber (Silver Halide); Sampling 2000-650 at $8 \mathrm{~cm}^{-1}$ resolution; Scan option: auto select, gain $1 \mathrm{X}$. 


\section{X-ray crystallography}

Single-crystal diffraction experiments were conducted on a Bruker APEX-II CCD diffractometer (13,14, 15b, 29 and 30) and an Xcalibur Sapphire3 diffractomer (19), using Mo Ka radiation. Crystals were cooled using Cryostream (Oxford Cryosystems) open-flow $\mathrm{N}_{2}$ cryostats. The structures were solved within Olex 2 by direct methods and refined by fullmatrix least squares against $\mathrm{F}^{2}$ of all data, using SHELXTL software [33-37]. All non-hydrogen atoms were refined anisotropically. Hydrogen atom positions were calculated geometrically and refined using the riding model. CCDC (1433617-1433622) contains the supplementary crystallographic data for this paper. The data can be obtained free of charge from The Cambridge Crystallographic Data Centre via http://www.ccdc.cam.ac.uk/getstructures.

\section{Supporting Information}

Supporting information features full synthetic procedures, in situ FTIR data plots, copies of ${ }^{1} \mathrm{H},{ }^{13} \mathrm{C}$ and ${ }^{11} \mathrm{~B}$ NMR spectra, and full crystallographic data including images and CIF files.

\section{Supporting Information File 1}

Full synthetic procedures, in situ FTIR data plots, and copies of ${ }^{1} \mathrm{H},{ }^{13} \mathrm{C}$ and ${ }^{11} \mathrm{~B}$ NMR spectra.

[http://www.beilstein-journals.org/bjoc/content/ supplementary/1860-5397-12-174-S1.pdf]

\section{Supporting Information File 2}

Crystallographic Information Files of compounds 13, 14,

15b, 19, 29, and 30.

[http://www.beilstein-journals.org/bjoc/content/ supplementary/1860-5397-12-174-S2.zip]

\section{Acknowledgements}

DRC thanks the EPSRC, BBSRC and High Force Research Ltd. for doctoral funding. GLZ also thanks the EPSRC and High Force Research Ltd. for doctoral funding. We thank Helen Osborne and Prof. Todd B. Marder for helpful discussions, Dr. Horst Puschmann for general crystallographic guidance and for assistance in the solution of the crystal structure of $\mathbf{1 9}$, and Dr. Dmitry S. Yufit for general crystallographic guidance.

\section{References}

1. Sridharan, V.; Suryavanshi, P. A.; Menéndez, J. C. Chem. Rev. 2011, 111, 7157-7259. doi:10.1021/cr100307m

2. Wang, Z.; Li, S.; Yu, B.; Wu, H.; Wang, Y.; Sun, X. J. Org. Chem. 2012, 77, 8615-8620. doi:10.1021/jo301560w
3. Li, H. Y.; Horn, J.; Campbell, A.; House, D.; Nelson, A.; Marsden, S. P. Chem. Commun. 2014, 50, 10222-10224. doi:10.1039/C4CC04940C

4. Akiyama, T.; Morita, H.; Fuchibe, K. J. Am. Chem. Soc. 2006, 128, 13070-13071. doi:10.1021/ja064676r

5. Cheng, X.; Xia, Y.; Wei, H.; Xu, B.; Zhang, C.; Li, Y.; Qian, G.; Zhang, X.; Li, K.; Li, W. Eur. J. Org. Chem. 2008, 1929-1936. doi:10.1002/ejoc.200701080

6. Kamino, S.; Murakami, M.; Tanioka, M.; Shirasaki, Y.; Watanabe, K.; Horigome, J.; Ooyama, Y.; Enomoto, S. Org. Lett. 2014, 16, 258-261. doi:10.1021/ol403262x

7. Benbrook, D. M.; Madler, M. M.; Spruce, L. W.; Birckbichler, P. J.; Nelson, E. C.; Subramanian, S.; Weerasekare, G. M.; Gale, J. B.; Patterson, M. K., Jr.; Wang, B.; Wang, W.; Lu, S.; Rowland, T. C.; DiSivestro, P.; Lindamood, C., III; Hill, D. L.; Berlin, K. D. J. Med. Chem. 1997, 40, 3567-3583. doi:10.1021/jm970196m

8. Dhar, A.; Liu, S.; Klucik, J.; Berlin, K. D.; Madler, M. M.; Lu, S.; Ivey, R. T.; Zacheis, D.; Brown, C. W.; Nelson, E. C.; Birckbichler, P. J.; Benbrook, D. M. J. Med. Chem. 1999, 42, 3602-3614. doi:10.1021/jm9900974

9. Atwal, K. S.; Ferrara, F. N.; Ding, C. Z.; Grover, G. J.; Sleph, P. G.; Dzwonczyk, S.; Baird, A. J.; Normandin, D. E. J. Med. Chem. 1996, 39, 304-313. doi:10.1021/jm950646f

10. Sánchez, I.; Pujol, M. D. Synthesis 2006, 1823-1828. doi:10.1055/s-2006-942360

11. Birch, A. J.; Lehman, P. G. J. Chem. Soc., Perkin Trans. 1 1973, 2754-2759. doi:10.1039/P19730002754

12. Smith, W. H.; Bard, A. J. J. Am. Chem. Soc. 1975, 97, 6491-6495. doi:10.1021/ja00855a034

13. Li, Y.-M.; Shen, Y.; Chang, K.-J.; Yang, S.-D. Tetrahedron Lett. 2014, 55, 2119-2122. doi:10.1016/j.tetlet.2014.02.043

14. Barnard, J. H.; Zhou, G.-L.; Collings, J. C.; Whiting, A.; Marder, T. B. Durham University, Durham, UK, Unpublished work.

15. Koo, J. J. Am. Chem. Soc. 1953, 75, 1891-1895. doi:10.1021/ja01104a034

16. Monnereau, C.; Blart, E.; Odobel, F. Tetrahedron Lett. 2005, 46, 5421-5423. doi:10.1016/j.tetlet.2005.05.117

17. Emmanuvel, L.; Shukla, R. K.; Sudalai, A.; Gurunath, S.; Sivaram, S. Tetrahedron Lett. 2006, 47, 4793-4796. doi:10.1016/j.tetlet.2006.05.062

18. Kawade, R. K.; Huple, D. B.; Lin, R.-J.; Liu, R.-S. Chem. Commun. 2015, 51, 6625-6628. doi:10.1039/C5CC01287B

19. Tomcufcik, A. S.; Seeger, D. R. J. Org. Chem. 1961, 26, 3351-3356. doi:10.1021/jo01067a079

20. Castanet, A.-S.; Colobert, F.; Broutin, P.-E. Tetrahedron Lett. 2002, 43, 5047-5048. doi:10.1016/S0040-4039(02)01010-9

21. Adimurthy, S.; Ramachandraiah, G.; Ghosh, P. K.; Bedekar, A. V. Tetrahedron Lett. 2003, 44, 5099-5101. doi:10.1016/S0040-4039(03)01144-4

22. Yamamoto, T.; Toyota, K.; Morita, N. Tetrahedron Lett. 2010, 51, 1364-1366. doi:10.1016/j.tetlet.2009.12.125

23. Klapars, A.; Buchwald, S. L. J. Am. Chem. Soc. 2002, 124, 14844-14845. doi:10.1021/ja028865v

24. Thompson, M. D.; Berlin, K. D. Proc. Okla. Acad. Sci. 1985, 65, 39-43.

25. Phippen, C. B. W.; Beattie, J. K.; McErlean, C. S. P. Chem. Commun. 2010, 46, 8234-8236. doi:10.1039/c0cc02502j

26. De, K.; Legros, J.; Crousse, B.; Bonnet-Delpon, D. J. Org. Chem. 2009, 74, 6260-6265. doi:10.1021/jo9012699

27. Reist, E. J.; Hamlow, H. P.; Junga, I. G.; Silverstein, R. M.; Baker, B. R. J. Org. Chem. 1960, 25, 1368-1378. doi:10.1021/jo01078a021

28. Spartan'10; Wavefunction, Inc.: Irvine, CA, 2010. 
29. Pettersen, E. F.; Goddard, T. D.; Huang, C. C.; Couch, G. S.;

Greenblatt, D. M.; Meng, E. C.; Ferrin, T. E. J. Comput. Chem. 2004, 25, 1605-1612. doi:10.1002/jcc.20084

30. Winterfeldt, E. Synthesis 1975, 617-630. doi:10.1055/s-1975-23856

31. Stevens, R. V.; Mehra, R. K.; Zimmerman, R. L. J. Chem. Soc. D 1969, 877-878. doi:10.1039/c29690000877

32. Ishiyama, T.; Murata, M.; Miyaura, N. J. Org. Chem. 1995, 60, 7508-7510. doi:10.1021/jo00128a024

33. Dolomanov, O. V.; Bourhis, L. J.; Gildea, R. J.; Howard, J. A. K.; Puschmann, H. J. Appl. Crystallogr. 2009, 42, 339-341. doi:10.1107/S0021889808042726

34. Sheldrick, G. M. Acta Crystallogr., Sect. A: Found. Adv. 2015, 71, 3-8. doi:10.1107/S2053273314026370

35. Sheldrick, G. M. Acta Crystallogr., Sect. C: Struct. Chem. 2015, 71, 3-8. doi:10.1107/S2053229614024218

36. Sheldrick, G. M. Acta Crystallogr., Sect. A: Found. Crystallogr. 2008, 64, 112-122. doi:10.1107/S0108767307043930

37. APEX2; SAINT; SADABS; Bruker AXS, Inc.: Madison, WI, 2014.

\section{License and Terms}

This is an Open Access article under the terms of the Creative Commons Attribution License (http://creativecommons.org/licenses/by/4.0), which permits unrestricted use, distribution, and reproduction in any medium, provided the original work is properly cited.

The license is subject to the Beilstein Journal of Organic Chemistry terms and conditions:

(http://www.beilstein-journals.org/bjoc)

The definitive version of this article is the electronic one which can be found at: doi:10.3762/bjoc. 12.174 